\title{
Trabajo de cuidado: mercantilización y desvalorización ${ }^{*}$
}

DOI: https://doi.org/10.18046/recs.iEspecial.3218

Care Work: Commodification and Devaluation

Javier A. Pineda D. ${ }^{* *}$

Universidad de los Andes (Bogotá, Colombia)

\footnotetext{
* El presente artículo es producto de un proyecto de profundización temática del autor, con base en proyectos anteriores de investigación sobre la organización social del cuidado en Colombia y el cuidado de la vejez en una sociedad en envejecimiento, cuyos resultados se han publicado parcialmente en Pineda (2011; 2014; 2018). Estos han contado especialmente con el apoyo del CIDER de la Universidad de los Andes. Artículo de investigación recibido el 31.10.2018 y aceptado el 08.02.2019.

${ }^{* *}$ Economista de la Universidad del Valle (Colombia) con Maestría en Economía del CIDE (México). Doctor en Estudios sobre Desarrollo por la Universidad de Durham (Inglaterra). Profesor asociado del Centro Interdisciplinario de Estudios sobre Desarrollo (CIDER) de la Universidad de los Andes (Colombia), desde el 2005 hasta la fecha. Correo electrónico: jpineda@uniandes.edu.co ORCID: https://orcid.org/oooo-ooo3 1183-7677
} 


\section{Cómo citar/How to cite}

Pineda D., Javier A. (2019). Trabajo de cuidado: mercantilización y desvalorización.

Revista CS, núm. especial, 111-136. https://doi.org/10.18046/recs.iEspecial.3218 
Resumen

Abstract

Basado en el giro conceptual del cuidado que se ha dado en la última década en América Latina, este artículo presenta, para el caso colombiano, la creciente mercantilización de actividades de cuidado y argumenta que dicho proceso no solo ha sido altamente feminizado, sino que también ha conllevado nuevas formas de desvalorización del trabajo de cuidado y femenino. Este proceso se evidencia de manera general para el servicio doméstico de cuidado remunerado en la familia y, de manera específica, para el trabajo de cuidado institucionalizado de la vejez, con base en fuentes tanto cuantitativas como cualitativas. Se argumenta entonces que, a pesar de los cambios en las modalidades de la prestación del servicio doméstico y del avance en la profesionalización del cuidado institucionalizado de la vejez, la mercantilización ha mantenido una precarización y desvalorización del trabajo de las cuidadoras, lo cual, a su vez, afecta el ejercicio y desarrollo de la ética del cuidado.

PALABRAS CLAVE:

trabajo de cuidado, mercantilización del cuidado, cuidado de la vejez, ética del cuidado

Based on the conceptual turn of care that has taken place in the last decade in Latin America, this article presents, for the Colombian case, the increasing commodification of care activities and argues that this process has not only been highly feminized but has also led to new forms of devaluation of care and women's work. In general, this process is evidenced in the domestic service of paid care in family and, specifically, in the work of institutionalized care of the elderly, based on both quantitative and qualitative sources. It is argued that despite changes in the modalities of domestic service provision and the advance in the professionalization of the institutionalized care of elderly, the commodification has led to a devaluation of the work of caregivers, which in turn affects the exercise and development of the ethics of care.

\section{KEYWORDS:}

Care Work, Commodification of Care, Elderly Care, Ethics of Care 



\section{Introducción}

La modernidad, como proyecto cultural o conjunto de ideas y valores que pretenden valer en sí y por sí mismos, se afianzó no solo en los valores de la razón, el universalismo, la homogeneidad, el progreso y el orden, sino también en el ser humano como centro del universo (antropocentrismo) y, especialmente, en el androcentrismo, al ubicar al varón como representante de lo humano (Hissong, 1996). Los procesos de modernización -la puesta en marcha de estos valores a través de la historia- excluyeron y negaron la ciudadanía a aquellas personas diferentes al varón blanco y propietario y, en especial, a las mujeres y lo femenino. La modernidad y los procesos de modernización reconfiguraron el poder de los hombres y afianzaron la dualidad entre los sexos, subordinando a las mujeres y lo femenino al campo de lo privado, por fuera del mercado.

Así, la asignación histórica de las mujeres a espacios privados y su exclusión de lo público las privó de los derechos ciudadanos, incluido el voto, la propiedad, el trabajo, la educación y el mercado. Las actividades llevadas a cabo en el espacio privado, ligadas a la reproducción de lo humano mismo, al no entrar en el intercambio en la esfera de los mercados, fueron negadas como trabajo y consideradas de índole natural. El trabajo reproductivo y de cuidado, como actividades propias del sexo femenino, fueron subvaloradas y subordinadas, al igual que las personas que las han desempeñado (Carrasco; Borderías; Torns, 2011).

No obstante, los procesos de modernización, con la constante necesidad de ampliar los mercados, han llevado no solo a sacar a la mujer del espacio de lo doméstico para expandir la oferta de trabajo en los mercados laborales, sino que también, paralelamente, han socializado y mercantilizado muchas actividades antes realizadas en el contexto de lo doméstico y de los cuidados en el hogar. Así, se han producido tres fenómenos paralelos: primero, una creciente participación de la mujer en los mercados laborales; segundo, una creciente socialización de algunos campos del cuidado; y, tercero, una creciente mercantilización de trabajos de cuidado.

En América Latina, este proceso de modernización se ha vivido de manera creciente a partir de la década de los setenta del siglo pasado, y continua en el presente siglo. Esto ha permitido una creciente lucha por reivindicar el cuidado como un trabajo y la búsqueda por develar la subordinación y valorar los cuidados y lo femenino, tanto en la esfera de lo doméstico como en lo público y el mercado (Arango; Molinier, 2011; Hirata; Araujo, 2012).

Los estudios sobre el cuidado han mostrado cómo las tareas que tradicionalmente han ocurrido en el ámbito doméstico son cruciales e imprescindibles para el funcionamiento del sistema económico y para el bienestar social (Daly; Lewis, 200o). Así, 
uno de los primeros procesos de modernización ha consistido en socializar, mediante una mayor presencia del Estado, algunas actividades de cuidado, particularmente aquellas referidas a la educación, la salud y la protección social, las cuales han tenido su propia dinámica desde la constitución de los Estados modernos. No obstante, más recientemente, a partir de finales del siglo $\mathrm{XX}$, se ha disputado la mayor presencia del Estado en la socialización del cuidado de la infancia, la vejez, la discapacidad y otros grupos que demandan cuidados especiales (Arriagada, 2013; Batthyány, 2015).

Este texto presenta, para el caso colombiano, la creciente mercantilización de actividades de cuidado y argumenta que dicho proceso no solo ha sido altamente feminizado, sino que también ha conllevado nuevas formas de desvalorización del trabajo de cuidado y femenino. Este proceso se evidencia, de manera general, para el servicio doméstico de cuidado remunerado en la familia y, de manera específica, para el trabajo de cuidado institucionalizado de la vejez, con base en fuentes cuantitativas recientes y trabajos de campo cualitativos previos del autor (Pineda, 2014). Se argumenta entonces que, a pesar del avance en los procesos de profesionalización del cuidado institucionalizado de la vejez, la mercantilización ha conllevado una precarización y desvalorización del trabajo de las cuidadoras, lo cual, a su vez, afecta el ejercicio y desarrollo de la ética del cuidado.

En la siguiente sección, se presentan algunos elementos conceptuales en el giro conceptual del cuidado y el florecimiento de los estudios sobre el trabajo de cuidado en América Latina. La tercera sección, evidencia el argumento general de la continuidad en la desvalorización del cuidado en el campo de lo doméstico y familiar. En la cuarta sección, se sostienen los principales argumentos alrededor del trabajo de cuidado en la vejez y, luego, la relación entre la desvalorización de este trabajo y la ética del cuidado. Finalmente, se cierra con algunas conclusiones.

\section{El giro conceptual del cuidado}

Desde la década de los setenta, lo que posteriormente se denominaría el feminismo de la igualdad -liberal o socialista-, muy centrado en el análisis de la división sexual del trabajo, señaló la importancia de la distinción entre trabajo productivo y reproductivo (Benería, 1979). Como muy bien lo señalaría Lourdes Benería (2006) casi tres décadas después, "el objetivo principal de esta distinción era poner de manifiesto la invisibilidad del trabajo de las mujeres y su concentración en la esfera reproductiva y no remunerada" (9).

Desde entonces, una oleada de estudios desde distintas disciplinas y enfoques analizaron el trabajo no remunerado de las mujeres en la esfera de lo doméstico, 
y sus múltiples efectos en las condiciones laborales, en un contexto de creciente participación laboral femenina. Uno de los primeros efectos a nivel conceptual fue la crítica feminista a los conceptos modernos de trabajo y trabajador. Dicha crítica develó su carácter androcéntrico, construido, teóricamente, desde la experiencia específica masculina que lo convirtió en norma universal, invisibilizando otras formas de trabajo, especialmente las actividades no remuneradas de las mujeres. Joan Scott (1993) señala cómo en los procesos de modernización europeos del siglo XIX se consolidó no solo la separación entre familia y trabajo, reproducción y producción, sino también la concepción del trabajo doméstico como una cualidad innata, propia de las mujeres como destino natural.

La crítica feminista al mundo del trabajo presenta distintas fuentes disciplinarias y contribuciones conceptuales, que han llevado, en las últimas décadas, al "giro conceptual del cuidado". Por un lado, se sitúan los enfoques que desde la división sexual del trabajo hicieron contribuciones en la sociología, con la tradición materialista en torno al modo de producción doméstico y el análisis socioeconómico del patriarcado (Delphy, 1982); en la antropología, el carácter cultural y situado del trabajo en sociedades premodernas (Comas, 1995); y en la historia, con la construcción moderna de la ideología de la domesticidad (Scott, 1993).

Por otro lado, la economía feminista anglosajona desarrolló, en la década de los noventa, una crítica a las teorías económicas predominantes, en la forma de entender los circuitos económicos exclusivamente desde lo que se involucraba en los mercados. La no consideración de todo aquello por fuera del mercado no solo impedía entender la dinámica económica misma, sino que obstruía el desarrollo económico, el bienestar social y constituía un sesgo masculino que invisibilizaba el aporte económico del trabajo de las mujeres (Elson, 1995; Folbre, 1994; 1995). Elson (1995) señala que el sesgo masculino en los procesos de desarrollo lleva a considerar a los hogares solo como unidades de consumo y no como productores de insumos y recursos valorables para el funcionamiento del sistema económico. Estas contribuciones dieron sustento a lo que se llamaría la economía del cuidado (care economy) que se define como el trabajo realizado y valor creado, primordialmente en la esfera doméstica (England, 2005).

Por su parte, una tercera tradición feminista, que va a introducir de manera más explícita el concepto de cuidado, es aquella que proviene de la psicología y la filosofía moral. El trabajo de Carol Gilligan (1982) abrió una agenda de estudios, especialmente en el feminismo norteamericano, para analizar las características emocionales y éticas del trabajo de las mujeres -principalmente el trabajo de cuidado directo de personas-, y cuestionar las teorías positivistas, racionalistas e individualistas del trabajo. La noción de "ética del cuidado", acuñada por Gilligan en su crítica a la teoría 
moral de Kohlberg, abrió también la discusión para situar el concepto de cuidado más allá de los ámbitos de los hogares y ubicarlo en el centro de la organización social, con múltiples repercusiones económicas y políticas (Tronto, 2013).

Joan Tronto (2013) desarrolla una crítica a la concepción liberal que prioriza el mercado como sistema adecuado para distribuir recursos desde la lógica de agentes racionales que persiguen su propio interés. Señala que los mercados se construyen bajo arreglos y relaciones de poder entre quienes proveen cuidado dentro y fuera del mercado, y quienes reciben estos cuidados, pero no se preocupa por la desvalorización de los servicios de cuidado y su calidad ni de la forma diferencial en que los ciudadanos acceden a estos. La autora señala la necesidad de avanzar hacia una "democracia cuidadora" que visibilice y abra la discusión sobre las responsabilidades del cuidado en la sociedad, y considere a quienes, por razones de clase, raza o género, están eximidos de dicha responsabilidad.

Las distintas fuentes que contribuyen al giro conceptual del cuidado hacen de este un concepto multidimensional e interdisciplinario. Así, la perspectiva del cuidado, en sus aportes y dimensiones éticas, subjetivas, emocionales, corporales e identitarias, se suman a los análisis en términos de la división sexual del trabajo. Luz Gabriela Arango (2015) rescata estas dimensiones, pero, además, enfatiza el cuidado como trabajo. Al señalar que el cuidado comprende disposiciones personales que remiten a la ética del cuidado, que generalmente se asocian con el amor y con la preocupación por el bienestar de otras personas, el cuidado es, ante todo, un conjunto de actividades físicas y emocionales que se deben realizar para atender necesidades y brindar bienestar a otras personas. Para estos efectos, menciona el trabajo de Pascale Molinier (2011), quien resalta el trabajo inherente al cuidado:

cuidar a otro no es pensar en el otro o preocuparse por él de manera intelectual e incluso afectiva; tampoco es necesariamente quererlo: es hacer algo, producir un determinado trabajo que participe directamente del mantenimiento o de la preservación de la vida del otro, es ayudarlo o asistirlo en las necesidades primordiales como comer, asearse, descansar, dormir, sentir seguridad y dedicarse a sus propios intereses (49).

El enfoque de la psicodinámica del trabajo, impulsado por Molinier, busca articular varias de las fuentes anteriores del giro conceptual del cuidado, debatiendo con las corrientes de la filosofía moral y feministas norteamericanas en torno a la ética del cuidado. En esta articulación, pone énfasis en el cuidado como trabajo, en las relaciones sociales y de poder en que se desenvuelve, en las condiciones culturales -pero también materiales- en las que surge y opera una ética del cuidado. No obstante, el aporte de la psicodinámica del trabajo está en el estudio de los mecanismos 
subjetivos que activan las mujeres para defenderse del sufrimiento y humillación que este tipo de trabajos pueden generar. En palabras de Arango (2015) se explicita con precisión este aporte:

Como lo analiza Molinier (2005), la experiencia concreta del cuidado genera malestar por la invisibilidad que caracteriza su éxito porque este debe borrarse como trabajo, anticipar la demanda y disimular los esfuerzos realizados para obtener el resultado. En esto reside el "saber-hacer discreto" que caracteriza al trabajo de cuidado, trabajo que solo se ve cuando falla.

Molinier y Paperman [2013] cuestionan el divorcio existente entre el análisis del trabajo de cuidado y la ética del cuidado debido a una concepción de la ética como un saber intelectual separado de las prácticas ordinarias. Este desinterés es explicado por Tronto en términos de "indiferencia de los privilegiados"; los cuales se benefician con el trabajo de cuidado de los grupos subalternos y no imaginan que sus prácticas puedan ser portadoras de dimensiones éticas (104).

El trabajo de cuidado se ha explorado desde un concepto ampliado que incorpora la producción feminista, incluyendo el concepto de trabajo emocional a partir del aporte seminal de Hochschild (1983), como las distintas fuentes sobre la ética del care (Paperman, 2011). Asimismo, el trabajo del cuidado se ha ampliado también con el concepto de trabajo corporal, entendido como aquel que implica un contacto directo con el cuerpo del otro (aseo, traslado, masaje, etc.), al tiempo que se realiza un trabajo emocional. El trabajo corporal y subjetivo con personas dependientes requiere que las trabajadoras manejen sus propios sentimientos con relación a la corporalidad de su trabajo, en la medida en que despliegan su labor de cuidado. La labor corporal supone un trabajo físico, en el cual el cuerpo es sujeto del trabajo, a la vez que sitio u objeto sobre el que los servicios son realizados (Adkins; Lury, 200o; Blood, 2005; Kang, 2010; McDowell, 2009).

Para cerrar esta sección, finalmente, se hará referencia a lo que se conoce como la organización social de los cuidados. ${ }^{1}$ La literatura anglosajona introdujo el concepto de cuidado social a partir del trabajo de Daly y Lewis (2000), quienes vinculan el trabajo de cuidado con los regímenes de bienestar y la política social. Esto facilita entender los marcos normativos donde se realiza el cuidado; es decir, las obligaciones y responsabilidades que asigna a los actores, como también vincular la medición del uso del tiempo y los costos económicos de las actividades de cuidado. De esta

1. Se utiliza el término en plural debido a que los cuidados se configuran en diferentes arreglos institucionales, políticas públicas y campos de disputa entre los distintos actores. 
manera, se abre el análisis a la forma como se distribuyen los cuidados entre los distintos agentes proveedores en la sociedad. Así, se establece la figura del "diamante de cuidado"; es decir, el régimen de cuidado que resulta de la interrelación entre el Estado, el mercado, las familias y la comunidad (Razavi, 2007).

Para América Latina, se han iniciado recientemente una serie de estudios sobre la organización social de los cuidados, mostrando la gran heterogeneidad entre países y entre los distintos campos de cuidado en cada uno de ellos, así como las profundas inequidades de género que subsisten en los mismos (Batthyány, 2018; Esquivel; Faur; Jelin, 2012; Vega; Gutiérrez, 2014). Al igual que en otros países de la región, en Colombia el trabajo de cuidado ha recaído sobre grupos específicos de mujeres, y ha obedecido a las transformaciones de la división sexual del trabajo, a la segmentación del mercado laboral por género y a las construcciones sociales de la diferencia de clase, género y étnico-raciales (Arango, 2010; Pineda, 2011).

Este artículo busca contribuir al análisis de la organización social de los cuidados en Colombia, desde el punto de vista de los procesos de mercantilización; es decir, de la mayor participación del mercado en ellos, para argumentar cómo estos procesos desvalorizan el trabajo de las cuidadoras.

\section{Desvalorización del trabajo de cuidado en la familia}

A partir de la idea de que no hay una organización social del cuidado a nivel nacional, sino un grupo heterogéneo de "diamantes de cuidado", entendido como diversas configuraciones interrelacionadas de arreglos institucionales, políticas públicas y "campos" de disputa entre los distintos actores ${ }^{2}$, en este apartado se presentará el campo del trabajo de cuidado en la familia, en donde este trabajo permanece desvalorizado.

La familia ha sido el ámbito histórico por excelencia donde se ha llevado a cabo el trabajo de cuidado. América Latina ha sido identificada como un sistema familista en la organización social de los cuidados, toda vez que es en la familia y, especialmente, en las mujeres a su interior sobre quienes ha recaído el cuidado de todos los miembros de la familia, con distintos grados de dependencia e interdependencia. Así, el cuidado no ha estado limitado solo a las personas con mayor demanda de él, o con diverso tipo o grado de dependencia, como la infancia, la vejez, la enfermedad o la invalidez, sino también a las personas adultas en condiciones de salud y

2. Se utiliza aquí el concepto propuesto por Bourdieu (1979), concebido como ese espacio social donde fracciones de clase disputan y ponen en juego determinados recursos o capitales específicos, tanto materiales como simbólicos, que definen y son definidos por normas sociales de relacionamiento y dominación, que configuran sus condiciones de vida. 
habilidad que, en la mayoría de los casos, tienen este privilegio en su condición de género, clase, edad o raza. Esta cualidad ostentada por sujetos de cuidado se refiere, principalmente, a varones de distintas clases sociales y de diferentes edades, que les permite participar en mejores condiciones en el mercado de trabajo; pero también por mujeres de clases medias o altas, especialmente jóvenes. No obstante, las personas adultas en las familias se trazan en una compleja interdependencia que mantiene profundas inequidades de género, intergeneracionales, de clase y de raza.

Dos grupos de mujeres cargan la mayor parte del trabajo de cuidado al interior de las familias. Primero, las amas de casa que, por razones de clase, edad y género, soportan el trabajo de cuidado como actividad principal y han sido sistemáticamente excluidas del mercado laboral. Segundo, las empleadas del servicio doméstico, quienes sostienen el cuidado en hogares de sectores altos y medios, y han soportado la continuidad histórica neocolonial de subordinación por razones de raza, clase y género, y, en las últimas décadas, el destierro y la violencia producto del conflicto armado.

El primer grupo expresa la discriminación de género en las sociedades contemporáneas en América Latina, y es la forma como la división sexual del trabajo mantiene a un grupo significativo de mujeres por fuera del mercado laboral remunerado. Así, el más importante grupo de mujeres sobre el que recae el trabajo de cuidado es aquel constituido por las "amas de casa", que eufemísticamente se sigue clasificando en las estadísticas como "población económicamente inactiva". ${ }^{3}$ Este componente, constituido por cerca de un tercio de las mujeres en edad de trabajar, está configurado por el orden de género en la sociedad, la dinámica sociodemográfica y los mercados laborales, que les niegan la entrada al mercado del trabajo remunerado.

La reducción de las brechas de género, a partir del incremento sostenido de la participación femenina en el trabajo productivo, observadas en las últimas dos décadas del siglo anterior, llevó a que, a finales del siglo XX, más de la mitad de las mujeres en edad de trabajar lo hiciera en forma remunerada y, en su mayoría, fuera de casa. Si bien esto constituyó una evidencia contundente de que amplios grupos de mujeres habían dejado de estar confinadas exclusivamente al trabajo reproductivo y doméstico, esta veloz transformación no solo ocultó la gran contradicción de lo que se ha denominado la doble jornada laboral femenina ${ }^{4}$, sino también la

3. Según la definición oficial del DANE, la población en edad de trabajar (PET) se divide entre la población económicamente activa (PEA) y la población económicamente inactiva (PEI). Estaúltima está constituida por estudiantes, pensionados y amas de casa.

4. Ann Oakley (1974), investigadora británica, fue la primera en introducir el concepto de la doble jornada (double burden), posteriormente, Hochschild y Machung (1990) lo popularizaron con el término de "el segundo turno" (the second shift). 
resignificación y desvalorización de las labores de cuidado por el mercado, y por un Estado y un varón ausentes.

La caída de las tasas de fecundidad y el proceso de transición demográfica, la urbanización de la sociedad y la expansión de los servicios educativos, entre otros, han sido los factores asociados a la mayor participación de la mujer en el trabajo remunerado, a partir de los años setenta en Colombia (Chenery, 1986). Esta participación se ha presentado tanto en el mercado laboral propiamente (asalariado), como en la producción directa de bienes y servicios (trabajo independiente). La tasa global de participación femenina ${ }^{5}$ para las grandes áreas urbanas en Colombia mostró un ritmo de crecimiento cercano a un punto porcentual promedio anual, durante las décadas de los ochenta y noventa, al pasar de $37 \%$, en 1982, a $47 \%$, en 1992, y a $56 \%$, en el año 200o. En el presente siglo, la participación ha crecido a un ritmo más lento, hasta alcanzar el $60 \%$, reduciendo cuatro puntos la brecha frente a los hombres (de 19 a 15 puntos).$^{6}$

El inverso de la tasa global de participación laboral femenina es la tasa de inactividad, la que representa a las mujeres “amas de casa”. El techo de género para una mayor participación laboral femenina significa que un grupo importante de mujeres que pudiera trabajar en el mercado remunerado permanece en lo que se denomina estadísticamente la "población económicamente inactiva"; es decir, mujeres que no participan ni en el empleo formal ni en actividades informales, que trabajan exclusivamente en actividades no remuneradas y que participan ampliamente en la economía del cuidado. Según los datos del DANE, para junio de 2018, las mujeres económicamente inactivas eran 9 millones para el total nacional, de las cuales 2,6 millones estudiaban (29\%), 5,3 millones se dedicaban a los oficios del hogar (59\%) y un millón eran ancianas o incapacitadas (12\%). Es decir, que en Colombia 5,3 millones de mujeres se dedican a la economía del cuidado, al trabajo doméstico de cuidado no remunerado (TDCNR) en el interior de los hogares. Así, este grupo específico de cuidadoras pasó de ser el $34 \%$ de la PET al $26 \%$, en lo corrido de este siglo.

Según la primera Encuesta Nacional de Uso del Tiempo (ENUT), aplicada por el DANE entre 2012-2013, este grupo es el que mayor tiempo le dedica a TDCNR. Dentro de las mujeres que reportan realizar TDCNR, se establecieron tres rangos de intensidad de tiempo diario dedicado a este trabajo: primero, las cuidadoras a

5. La tasa de participación laboral es el porcentaje de la población en edad de trabajar (población mayor de 12 años) que es económicamente activa (PEA); es decir, que trabaja o busca trabajo remunerado.

6. La tasa global de participación para los hombres ha estado alrededor de $75 \%$ en el presente siglo.

7. Datos con base en encuestas de hogares del DANE: ECH (2001-2006) y GEIH (2007-2018), para 13 áreas metropolitanas. 
tiempo corto (menos de tres horas); segundo, las cuidadoras a medio tiempo (entre tres y seis horas); $\mathrm{y}$, tercero, las cuidadoras a tiempo completo (más de seis horas). Lo evidente es que este último grupo de mujeres constituye un porcentaje muy cercano al de las mujeres amas de casa ( $23 \%$ vs. $26 \%$ ), lo cual significa que la gran mayoría de mujeres amas de casa, consideradas "inactivas", se dedican al TDCNR de tiempo completo, con un tiempo promedio diario de ocho horas y media. ${ }^{8}$

Este grupo, por supuesto, es heterogéneo, pero, de acuerdo con la estratificación socioeconómica de los hogares, en su gran mayoría se trata de mujeres de hogares de estratos bajos que presentan una alta dependencia económica de otros miembros del hogar. El carácter de clase de esta discriminación de género supedita al $26 \%$ de las mujeres a la dedicación exclusiva del hogar y se expresa también en el nivel educativo de las amas de casa: $24 \%$ no alcanzan la educación básica primaria, $35 \%$ tienen educación básica primaria y $33 \%$ alcanzan la educación básica secundaria y media; solo el 4 \% tienen educación técnica y $3 \%$ educación universitaria. Es decir, el $93 \%$ son mujeres no calificadas.

El segundo grupo de mujeres que soporta el trabajo de cuidado al interior de las familias, lo constituyen las mujeres empleadas en el servicio doméstico remunerado. Este tipo de trabajo se ha destacado, históricamente, por sus características de servidumbre y explotación, que fueron ampliamente documentadas debido a su vertiginoso crecimiento en América Latina a partir de los procesos de migración masiva de mujeres campesinas en los años sesenta y setenta (León, 1993). No obstante, su caída a partir de los ochenta hizo afianzar la idea de que la universalización de la educación básica, la caída de la fecundidad y los procesos de modernización llevarían a su extinción o, en el peor de los casos, a la eliminación de su servidumbre y a la formalización de sus relaciones laborales.

Ciertamente, el porcentaje de trabajadoras de servicio doméstico, como proporción de la población femenina ocupada, ha caído significativamente. Este se redujo a la mitad cuando pasó de ser cerca del $16 \%$ de la población ocupada femenina, en 1984, al $8 \%$, en 1997. Sin embargo, con la crisis económica de finales de siglo, su participación subió de nuevo al nivel de principios de los noventa (13\%) en el año 2002, para descender, en el 2007, al mismo nivel de diez años antes y llegar al 6,5\% en el 2018. Esto significa que dicho empleo se configura históricamente y no obedece a una idea general de modernización y progreso que lleve a su extinción.

Por el contrario, a pesar de los esfuerzos de regulación especial, su presencia ha sido persistente, tanto en su tamaño (continúa ocupando en términos absolutos a cerca de 680 mil mujeres en las grandes áreas metropolitanas) como por el hecho 
de continuar siendo, en su gran mayoría, un empleo precario e informal, que desvaloriza el trabajo de cuidado de las mujeres a favor de los hogares de sectores de ingresos medios y altos. Las relaciones de servidumbre se continúan presentando como parte de los patrones culturales que discriminan por razones de clase, raza y género, que vinculan los trabajos de menor estatus con mujeres pobres o migrantes de grupos étnicos y que ven dichas actividades como vocación natural femenina. Aunque se ha reducido la participación del servicio doméstico de tipo interno-con residencia en el hogar de trabajo-, las necesidades de trabajo de muchas mujeres, en condiciones de desplazamiento forzado o no, y la debilidad de las instituciones laborales en Colombia han contribuido a mantener la precariedad de este trabajo de cuidado por excelencia.

Los estudios sobre desplazamiento forzado interno en el marco del conflicto armado, que comenzaron a producirse desde mediados de los noventa, documentaron tanto la feminización del desplazamiento -alto porcentaje de mujeres-como el restablecimiento laboral en los lugares de llegada mediante trabajos de servicio doméstico (Meertens, 2011). Sin duda, el masivo desplazamiento forzado en el campo colombiano que, en las últimas décadas, afectó a millones de habitantes, nutrió la oferta laboral femenina para el servicio doméstico. Si bien dicho efecto solo se observa en las estadísticas en el período de la crisis económica a finales de siglo y hasta $2002^{9}$, sí ha afectado la composición del servicio doméstico, en la medida en que todos los trabajos cualitativos, hasta la fecha, evidencian en el servicio doméstico una ocupación por excelencia de las víctimas del desplazamiento interno (ENS, 2018; Esguerra et al., 2018).

En esta línea, entre los trabajos de investigación más reconocidos, Jeanny Posso (2008) analiza en detalle la migración y el trabajo de servicio doméstico de mujeres inmigrantes negras procedentes del Litoral Pacífico colombiano en el mercado de trabajo de la ciudad de Cali. Este estudio reconoce que el servicio doméstico es quizás el más representativo para mujeres negras migrantes. La autora indaga los distintos tipos de desigualdad social marcados por la discriminación de género, clase y raza en el contexto laboral del occidente colombiano.

En los últimos años, en Colombia se ha avanzado normativamente en la protección de los derechos laborales de las trabajadoras del servicio doméstico. La aprobación del Convenio OIT 189 "Sobre el trabajo decente para las trabajadoras y los trabajadores domésticos", mediante la Ley 1595 de 2012, marcó un hito en el

9. Si bien la participación de servicio doméstico dentro de la población ocupada femenina ha caído al $6,5 \%$, el incremento en la modalidad de servicio por días permite atender a un número mayor de hogares. Aunque esta modalidad de servicio incrementa el ingreso de las trabajadoras, no solo intensifica su trabajo, sino que también dificulta el pago de prestaciones sociales. 
tema, que colaboró con la movilización y búsqueda de plenos derechos laborales. No obstante, un estudio reciente señala que este es:

Un oficio socialmente minusvalorado, invisibilizado y altamente vulnerable, no solo por su precariedad (el 61\% de estas personas gana menos de un salario mínimo, el 77\% recibe alimentos como pago en especie, y al 99\% no les pagan horas extras, para solo mencionar algunas cifras), sino también por estar cruzado de discriminaciones de todo tipo: de clase (la mayoría habita en cordones de pobreza de las ciudades y muchas son víctimas del conflicto armado), discriminación de género (el 98\% son mujeres), y el racismo (muchas son negras) (ENS, 2018: 2).

Como se puede observar, la coexistencia de los distintos tipos de discriminación y diferenciación social ha estado presente en los estudios sobre trabajo doméstico en Colombia; es decir, existe un esfuerzo por aplicar enfoques de interseccionalidad. Pero uno de los campos que comienza a indagarse es aquel de las relaciones entre patronas y patronos con las trabajadoras de servicio doméstico y, en alguna medida, las configuraciones éticas en ellas. Un estudio reciente, inspirado en el trabajo de Pascale Moliner (2011), indaga por las relaciones que establecen patrones hombres que viven solos con el trabajo de servicio doméstico; en este se encuentra una diversidad de experiencias que expresan continuidades y cambios en la división del trabajo, y en las relaciones de los varones frente al trabajo doméstico (Cuéllar, 2018).

\section{Mercantilización y desvalorización del cuidado de la vejez}

En el contexto de transición demográfica y aumento de la esperanza de vida, en América Latina la tasa de crecimiento de las personas mayores es entre tres y cinco veces más alta que la de la población total, del 2010 al 2025 y del 2025 al 2050. Como resultado, la proporción de personas mayores de 60 años se triplicará en ese período. Para Colombia, mientras en 1985 las personas mayores de 6o años representaban el $5 \%$ de la población, en 2015 su participación fue del $12 \%$ y, para 2025, será del $14 \%$ (CEPAL, 2005).

Este envejecimiento poblacional ha generado una gran demanda de cuidado de las personas mayores, pero las discusiones se centran, generalmente, en los sistemas de pensión, bajo el supuesto de que el cuidado descansa en la familia o las redes familiares. Sin embargo, este es un supuesto altamente problemático: primero, por la reducción del tamaño de las familias, su diversificación, los patrones de residencia en espacios más reducidos y los cambios en los papeles y relaciones de género (Arriagada, 2006; Pineda, 2010); segundo, porque no se puede asumir que la existencia 
de familiares sea una garantía de cuidado efectivo (Lloyd-Sherlock; Locke, 2008); y, tercero, porque más que la familia como unidad de análisis, el cuidado es altamente feminizado y descansa sobre las mujeres, quienes disponen de menor tiempo debido a su creciente participación en el mercado laboral (Pineda, 2011; Robles, 2006).

Si bien las mujeres en la familia continúan siendo la principal fuente de cuidado, el envejecimiento poblacional y la demanda de cuidado han propiciado la emergencia, desde el mercado, de una diversidad de establecimientos comerciales como casas de cuidado para ancianos o centros gerontológicos y geriátricos, dirigidos a familias de estratos medios y altos con capacidad de pago. Así, el proceso de envejecimiento ha reconfigurado el diamante de cuidado en el campo de la vejez, con una mayor participación del mercado. En otros textos, se ha analizado cómo este proceso de mercantilización del cuidado de la vejez se ha hecho mediante la institucionalización de los ancianos, lo cual ha llevado, por un lado, a la segregación o aislamiento de las personas mayores y, por otro, a la insostenibilidad de este modelo, y a la desvalorización de la vejez y la pérdida de su dignidad al final de sus días (Pineda, 2014; 2018). En este aparte, se mostrará cómo la mercantilización ha llevado a la desvalorización del trabajo de las cuidadoras.

En Bogotá, el promedio de registros mercantiles de establecimientos comerciales de cuidado de la vejez en la década de 1990 fue de ocho (8) por año, en la década de los 2000 fue de 26 , y el promedio entre 2010 y 2014 ha sido de $39 .{ }^{10}$ Teniendo en cuenta que muchas de estas casas no permanecen en el tiempo, para 2014 existía un total de 521 establecimientos con registro vigente. La encuesta representativa que se realizó en 144 de estas casas (Pineda; Guevara, 2014), mostró que, efectivamente, la mayoría de los centros gerontológicos encuestados $(55 \%)$ surgieron en forma creciente durante la segunda mitad de la década del 200o. En esta encuesta, se recogió también información organizacional sobre el personal contratado que labora en la institución, la distribución de horarios, las jornadas laborales, los cargos, capacitaciones ofrecidas, etc. Igualmente, se entrevistó a cuidadoras, al igual que a personal administrativo y personas mayores.

El cuidado de la vejez en los centros gerontológicos de Bogotá, descansa en dos grupos. Primero, están los auxiliares de enfermería, donde, de los contratados, el $85,2 \%$ son mujeres y $14,8 \%$, hombres. Asimismo, se contratan otros profesionales de la salud, como médicos, terapistas, etc., que, en su mayoría, siguen siendo mujeres (69\%), pero que, a diferencia de las auxiliares, son pocos y no son permanentes, pues trabajan solo algunos días a la semana o al mes. Segundo, está el personal de servicios generales, ocupación que es altamente demandada en todas las casas para 
los oficios de aseo y apoyo en el servicio de cuidado, y que, en un $80 \%$, son mujeres. En el área administrativa, especialmente para las casas más grandes, se contratan administradores y auxiliares de oficina, ocupaciones donde se encuentra la tasa más alta de hombres, con una participación del $40 \%$.

Estos dos grupos de cuidadoras tienen dos perfiles diferentes. Las auxiliares de enfermería, con formación técnica de dos años, en general son más jóvenes (66\% menor de 30 años). El personal de servicios generales, no calificadas, presenta mayores edades ( $72 \%$ mayor a 30 años) y, en muchos casos, han sido trabajadoras de servicio doméstico. Varios factores hacen que estos trabajos sean precarios. Primero, existe una muy alta inestabilidad, especialmente en las auxiliares de enfermería, relacionada con las precarias condiciones de trabajo-como se verá enseguida-frente a las expectativas de las trabajadoras. La principal preocupación de la gerencia o propietarios de los centros es, justamente, la alta rotación de las jóvenes auxiliares de enfermería. Esto, a su vez, mina la ética del cuidado, afecta la calidad del servicio y dificulta la vida digna de los ancianos (Pineda, 2018).

Las auxiliares de enfermería devengan salarios iguales a un mínimo y medio (63,7\%), al mínimo (21\%) o alrededor de dos salarios mínimos (10\%), y 5,6 \% devenga por debajo del salario mínimo. Las mujeres de servicios generales, quienes realizan los oficios más pesados de cuidado ${ }^{11}$, devengan el salario mínimo (67\%), el mínimo y medio ( $27 \%$ ) y un $5 \%$ está por debajo del mínimo. Con respecto a las jornadas laborales, se encontró que la mitad de las auxiliares (50\%) tienen turnos de 12 horas diarias; $21,7 \%$, de 8 horas; y 26,8 \%, de 24 horas. Generalmente, tienen jornadas de 48 horas semanales de trabajo, pero muchas laboran jornadas mayores a la legal.

Lo que se encuentra en muchas casas de cuidado, especialmente en las que atienden estratos medios-bajos y presentan dificultades de sostenibilidad financiera, es que tienen una nómina legal y otra informal, sin registro ni prestaciones o mediante empresas de intermediación. Esto se presenta para algunas auxiliares de enfermería, pero especialmente para cuidadoras de servicios generales, aunque estas tienen una mayor estabilidad. Se evidencian así condiciones laborales precarias en las cuidadoras, con alta inestabilidad y permanente rotación, horarios excesivos de trabajo, bajos salarios y baja calidad de vida. La desvalorización del cuidado se da así en un tipo de círculo vicioso, donde las condiciones precarias afectan la estabilidad y esta afecta la formalización; a su vez, la estabilidad perjudica el cuidado. Adicionalmente, muchas casas presentan limitados recursos logísticos y de infraestructura, así como

11. Estas cuidadoras generalmente realizan las tareas más pesadas y desvalorizadas de cuidado ("trabajo sucio"), como bañar a las personas mayores con mayor grado de dependencia, levantar, asear, etc., también con altos componentes emocionales y corporales. Por su carácter feminizado y no reconocido socialmente, su remuneración es injustamente menor. 
de equipos que faciliten la labor de cuidado, lo cual las enfrenta a una sobrecarga laboral y emocional. En las casas que enfrentan mayores dificultades, se evidencian las angustias de las trabajadoras para enfrentar las necesidades de los ancianos sin los recursos requeridos ni las condiciones mínimas para la atención, aparte de los atrasos en sus pagos y la atención de sus propias necesidades y el autocuidado de su salud.

El trabajo de cuidado suele presentar una sobrecarga laboral y emocional. Las tareas que realizan las cuidadoras requieren no solo los esfuerzos físicos comunes de bañar, transportar, alimentar, etc., a las personas mayores con niveles altos de dependencia, sino también grandes esfuerzos emocionales. Como se mencionó antes, el trabajo de cuidado es también un oficio subjetivo y emocional. En algunos establecimientos, se exige que la trabajadora deje sus problemas personales a un lado y se enfoque exclusivamente en el bienestar físico y emocional de su paciente. Además, son pocos los espacios en los que se atienden las necesidades emocionales y psicológicas de las cuidadoras. Los distintos estudios de caso han evidenciado que las y los cuidadores de personas mayores sufren impactos físicos y emocionales importantes que dependen de factores como las motivaciones, las exigencias del cuidado mismo, el involucramiento con la persona mayor, el tiempo que se lleva trabajando en esta área, así como la energía e intensidad que implique el cuidado. Otros estudios han evidenciado el síndrome de carga del cuidador (Caregiver Burden Syndrome) y los efectos en su calidad de vida (Martínez et al., 2009; Zambrano; Ceballos, 2007).

Como se argumentó en la anterior sección, el trabajo de cuidado no institucionalizado, en el ámbito familiar, suele ser no remunerado, desvalorizado y poco visible. En este aparte, se argumenta que, en el cuidado mercantilizado e institucionalizado, las condiciones laborales y el trabajo precario de las cuidadoras en los nuevos establecimientos de cuidado de la vejez, con bajos ingresos, largas jornadas y costos emocionales, contribuye a la desvalorización del trabajo de cuidado de miles de mujeres cuidadoras vinculadas a estos centros. Ahora bien, en cuanto a los efectos de esta desvalorización en la calidad del servicio de cuidado y su ética, es necesario entrar a la dimensión emocional del trabajo de cuidado, a partir especialmente de los testimonios de las cuidadoras.

\section{Trabajo emocional y ética del cuidado}

Para el cuidado de ancianos con un mayor grado de dependencia, el régimen de turnos y síndrome de carga de las cuidadoras se expresa en lo que algunas administradoras de los centros denominan la "falta de vocación" de las cuidadoras, en particular para las jóvenes auxiliares de enfermería. Pero qué es la "falta de vocación" y cuáles son 
sus causas son preguntas que invitan a deconstruir esta preocupación de la gerencia que centra la responsabilidad del cuidado en la "naturaleza" de la cuidadora.

La falta de vocación se asocia con los abusos que se cometen contra las personas mayores que requieren de cuidados. Estos abusos van desde sentarlos toda una jornada frente a un televisor, hasta esperar el cambio de turno para dejarle el aseo de un anciano de toda una noche a la cuidadora del turno siguiente.

El manejo en cama es difícil, sobre todo cuando ellos empiezan a tener una laceración, o sea a tener heridas, es terrible. Digamos, yo he llegado al turno de la mañana y encuentro que no le han realizado un cambio, no le han realizado una curación, una limpieza, es terrible. Hay ocasiones que es peor, ni se diga (Betulia, cuidadora, 49 años, 2013).

La falta de vocación es, entonces, la ausencia de lo que se llama desarrollo de una ética del cuidado. Esta actitud no es producto exclusivo de las condiciones laborales y el trabajo precario que se ha descrito, como tampoco es una característica propia del cuidado familiar. Como se verá, en condiciones limitadas muchas mujeres logran desarrollar una ética de cuidado y conservan la dignidad de las personas cuidadas. Pero, sin duda, las condiciones precarias de trabajo no contribuyen a las motivaciones y a las exigencias del cuidado mismo, que permitan el involucramiento con la persona mayor y el desarrollo de una responsabilidad del cuidado. Esta desmotivación de las jóvenes cuidadoras parece estar relacionada con la despersonalización que genera el no proyectar su vida profesional en estas organizaciones de cuidado. Su paso por estas "pequeñas empresas" constituye para ellas un trabajo "paraguas", mientras encuentran un empleo propio en una mejor entidad. "Aquí estoy porque es difícil encontrar un buen empleo en una clínica. Pero yo sigo buscando mientras tanto algo mejor, algo donde uno pueda proyectarse, como le digo, donde le paguen todo y uno esté mejor" (Jessica, cuidadora, 27 años).

Hablar del desarrollo de una ética del cuidado significa que esta no es una calidad innata propia de lo femenino, una cualidad "esencial" de las mujeres con "vocación" de cuidado. Ciertamente, está ligada al cultivo de valores en el nivel más profundo de la cultura, pero puede ser moldeada y desarrollada en distintos momentos de la vida de una persona por el contexto institucional y organizacional en el que se encuentre. Así, palabras que están en los testimonios de las cuidadoras, como paciencia, tolerancia, comprensión, amor, afecto, felicidad, etc., con un muy alto contenido de descripción emocional, presentan una alta frecuencia en la narrativa de la relación que ellas establecen en el proceso de cuidado de los ancianos y las ancianas. Es justamente esta narrativa la que se encuentra especialmente en mujeres que llevan un tiempo mayor de permanencia en las casas de cuidado, que han construido su proyecto de 
trabajo ahí, que han desarrollado una identidad como cuidadoras. Es en las mujeres de servicios generales en las que se evidencia, con mayor facilidad, la construcción de una ética del cuidado, un sentido de responsabilidad por el bienestar del otro. Son ellas las que hacen el trabajo "sucio" de cuidado, el más desvalorizado, las que sostienen la dignidad de las personas mayores, las que desarrollan una ética del cuidado.

Las palabras con las que se describe el cuidado se presentan también en un contexto de tensiones entre la identidad y el carácter propio de la cuidadora, y su función profesional y laboral de cuidado. Estas tensiones están relacionadas estrechamente con el carácter generalmente mixto del cuidado, entre lo público y lo privado, entre lo familiar e institucional, entre lo personal y lo profesional, entre el hogar y el mercado. La fuerza de estas dualidades modernas en conflicto suele ser resistida por la convergencia de las prácticas relacionales del cuidado, donde el trabajo emocional, aquel trabajo que produce un cambio subjetivo en quien recibe dicho cuidado, juega un papel central para el bienestar de las personas.

Hochschild (1983) considera que uno de los elementos que distingue el trabajo emocional es "la producción de un efecto subjetivo en el otro". En los testimonios de las cuidadoras se puede apreciar esto:

Yo me acostumbré a estar atenta a lo que requieran las viejitas, esa es mi vida, pues llevo aquí en esto un buen tiempo y siento que es con lo que me realizo, me siento bien. Cuando además de atenderlas les comparto algo, un cuento, alguna cosa y se ríen conmigo. Siento esa responsabilidad de que me necesitan, de poderlas atender, de que si lo hago lo hago con buena disposición; es que he aprendido que para ellas y para mí es mejor así. Ellas se sienten bien y yo soy feliz con eso (Mercedes, cuidadora, 58 años, 2014).

En este testimonio se expresa una producción emocional en la persona cuidada, cuando las viejitas "ríen conmigo", pero, además, hay una construcción de identidad de la cuidadora a partir de su ejercicio de cuidado y su subjetivación en la producción de un resultado, no en la persona cuidada, sino en la propia cuidadora: "siento que es con lo que me realizo", lo que enfatiza el carácter relacional del cuidado y el resultado intersubjetivo del trabajo emocional. En tal sentido, el trabajo también produce a la trabajadora subjetivamente.

Volviendo a las palabras del cuidado, en el anterior testimonio también se encuentra que la cuidadora dice que "he aprendido". Es decir, que la "vocación" no viene con ella, sino que se adquiere a partir de la interacción con las personas cuidadas. Los principios morales no surgen entonces de una predisposición innata, resultan de un cúmulo decantado de experiencias subjetivas aprendidas en distintos momentos del curso de vida, pero también de un ejercicio concreto de cuidado. 
Desde las reflexiones de la ética del cuidado, este trabajo resulta central para la humanización de las relaciones sociales (Arango; Molinier, 2011). Esto se aprecia en la potencialidad que tiene este tipo de labores de generar reflexividad sobre la posición del otro, la consideración por el otro y la experimentación del otro. Las reflexiones sobre la otredad hacen parte de la dimensión ética del trabajo y constituyen un elemento clave no solo para la reconceptualización del trabajo, sino también para la construcción de nuevas relaciones sociales de una manera mucho más amplia.

El trabajo, como espacio de construcción de identidades, continúa teniendo gran relevancia en la configuración de las identidades contemporáneas de las sociedades latinoamericanas. Pero este proceso de cambio de identidades también implica una transformación de actitudes, comportamientos y patrones de relacionamiento, a partir del contexto sociolaboral en el que se interactúa. El trabajo emocional en el cuidado lleva consigo también un proceso de autoevaluación o revalorización personal que hace parte de la ética misma que puede desarrollar el trabajo de cuidado (Soares, 2012).

\section{Conclusiones}

Los cambios ocurridos en las últimas décadas en Colombia han conllevado procesos de modernización que se continúan dando, de manera contradictoria, para el trabajo de miles de mujeres. Este artículo ha analizado dichos procesos para el caso del trabajo de cuidado en dos campos específicos: el cuidado de la familia y el cuidado de la vejez. En estos campos, se ha venido presentando una reconfiguración de lo que se denomina organización social de los cuidados, a partir, especialmente, de la dinámica del mercado laboral, así como de la mercantilización de los servicios de cuidado.

En el contexto de lo que se ha denominado en este texto el "giro conceptual del cuidado", analizar estos casos ha resultado relevante no solo por la validez de los conceptos que este giro ha integrado, sino también por la persistencia de las injusticias de género que motivaron los primeros esfuerzos conceptuales alrededor de la división sexual del trabajo. La subvaloración del trabajo femenino y feminizado de cuidado persiste, primero, en la forma más típica de la división entre las esferas pública y privada, que recluyen a una cuarta parte de las mujeres a trabajar en forma exclusiva como "amas de casa" en el trabajo doméstico de cuidado no remunerado.

En segundo lugar, se encuentra que la desvalorización del trabajo de cuidado también persiste en la continuidad del alto número de empleadas del servicio doméstico, un trabajo que simboliza las características de subordinación interpersonal de los trabajos premodernos herederos de la violencia colonial, racista, sexista y clasista. 
Tercero, la desvalorización del trabajo de cuidado se ha dado en claros procesos de mercantilización de servicios de cuidado, como ha sido el caso del cuidado de la vejez. Por un lado, para las auxiliares de enfermería, grupo principal de trabajadoras de cuidado en casas gerontológicas, no ha sido suficiente su profesionalización para evitar dicha desvalorización. Por otro lado, para las trabajadoras de servicios generales, quienes se encuentran en la base de la pirámide en la jerarquía ocupacional, y soportan el trabajo más pesado y "sucio" del cuidado.

Así, la creciente mercantilización de actividades de cuidado no solo ha sido altamente feminizada, sino que también ha conllevado nuevas formas de desvalorización del trabajo de cuidado y femenino. La distribución social del cuidado de la vejez ha recaído, tradicionalmente, sobre el trabajo no remunerado de las mujeres del hogar, bien sea hijas, compañeras o familiares. No obstante, los cambios demográficos y sociales han generado el surgimiento del cuidado institucionalizado que, para algunos sectores sociales, constituye una alternativa de cuidado, ante la reducción del tamaño de las familias y las demandas de tiempo y trabajo, especialmente en el contexto de la vida urbana. Estos nuevos espacios siguen siendo de cuidado femenino y parecen representar nuevas formas modernas de segregación y aislamiento de la vejez.

El potencial humanizador de la práctica del cuidado tiende a ser socavado por las condiciones laborales de las cuidadoras y los mercados de trabajo en que se insertan. La creciente demanda de cuidado para personas mayores en condiciones de dependencia ha llevado a la aparición de un creciente cuidado institucional y comercial desde el mercado, con alta segmentación de la oferta y con un posible deterioro de la calidad del mismo. El impulso que el mercado le da al trabajo de cuidado en un contexto de creciente demanda plantea serios cuestionamientos acerca de si el trabajo de cuidado en hogares de ancianos devalúa o no las emociones en favor de la eficiencia operativa y los beneficios.

\section{Referencias}

Adkins, Lisa; Lury, Celia (200o). Making bodies, making people, making work. En Organizing Bodies. Policy, Institutions and Work (pp. 151-165), editado por Linda McKie; Nick Watson. London: MacMillan Press.

Arango, Luz G. (2010). Género e identidad en el trabajo de cuidado. En Trabajo, identidady acción colectiva (pp. 81-107). México: UAM/CLACSO/Plaza y Valdés. 
Arango, Luz G. (2015). Cuidado, trabajo emocional y mercado: los servicios estéticos y corporales. Revista Latinoamericana de Estudios de Familia, 7, 99-120.

Arango, Luz; Molinier, Pascale (2011). El cuidado como ética y como trabajo. En El trabajo y la ética del cuidado (pp. 15-21). Medellín: La Carreta/Universidad Nacional de Colombia.

Arriagada, Irma (2006). Cambios de las políticas sociales: políticas de género y familia. Santiago de Chile: CEPAL.

Arriagada, Irma (2013). Desigualdades en la familia: trabajo y cuidado en Chile. En Desigualdad en Chile: La continua relevancia del género (pp. 91-112), editado por Claudia Mora. Santiago: Editorial Universidad Alberto Hurtado.

Batthyány, Karina (2015). Los tiempos del bienestar social. Género, trabajo no remunerado y cuidados en Uruguay. Montevideo: Inmujeres/MIDES.

Batthyány, Karina (2018). La organización social del cuidado. Políticas, desafíos y tensiones. En Género y cuidado: teorías escenarios y políticas (pp. 205-219), compilado por Luz Arango; Adira Amaya; Tania Pérez-Bustos; Javier Pineda. Bogotá: Universidad Nacional de Colombia/Universidad de los Andes/Universidad Pontificia Javeriana.

Benería, Lourdes (1979). Reproduction, Production and the Sexual Division of Labor. Cambridge Journal of Economics, 3(3), 203-225.

Benería, Lourdes (2006). Trabajo productivo/reproductivo, pobreza y políticas de conciliación. Nómadas, 24, 8-21.

Blood, Sylvia (2005). Body Work: The Social Construction of Women's Body Image. New York: Routledge.

Carrasco, Cristina; Borderías, Cristina; Teresa Torns (2011). El trabajo de cuidados. Historia, teoría y políticas. Madrid: Catarata.

Chenery, Hollis (1986). El problema laboral colombiano: diagnóstico, perspectivas y políticas. Informe final de la misión de empleo. Economía Colombiana, Serie de documentos, Separata N. ${ }^{\circ}$ 10, Contraloría General de la República, Bogotá.

Comas, Dolors (1995). Trabajo, género, cultura. Barcelona: Icaria/Institut Català d’Antropologia.

Comisión Económica para América Latina (2005). Dinámica demográfica y desarrollo en América Latina y el Caribe. Cuadernos de Población y Desarrollo, 58. Santiago de Chile.

Cuéllar, Priscila Victoria (2018). Contratando domesticidad. Estudio de caso de varones y su relación con lo doméstico (Tesis de maestría). Bogotá: Universidad Nacional de Colombia.

Daly, Mary; Lewis, Jane (2000). The concept of social care and the analysis of contemporary welfare states. British Journal of Sociology, 51(2), 281-298. 
Delphy, Christine (1982). Por un feminismo materialista: el enemigo principal y otros textos. Barcelona: La Sal.

Elson, Diane (1995). Male bias in the development process. Manchester/New York: Manchester University Press.

England, Paula (2005). Emerging Theories of Care Work. Annual Review of Sociology, 31, 381-399.

Escuela Nacional Sindical (2018). Historias tras las cortinas: cifras y testimonios sobre el trabajo doméstico en Colombia. Informe especial. Medellín: Escuela Nacional Sindical.

Esguerra-Muelle, Camila; Sepúlveda-Sanabria, Ivette; Fleischer, Friederike (2018). Se nos va el cuidado, se nos va la vida: migración, destierro, desplazamiento y cuidado en Colombia. Documentos de Política 3. Bogotá: CIDER Universidad de Los Andes.

Esquivel, Valeria; Faur, Eleonor; Jelin, Elizabeth (ed.) (2012). Las lógicas del cuidado infantil. Entre las familias, el Estado y el mercado. Buenos Aires: IDES/UNFPA/UNICEF.

Folbre, Nancy (1994). ¿Who Pays for the Kids? Gender and the Structures of Constraint. Nueva York: Routledge.

Folbre, Nancy (mayo, 1995). Engendering Economics: New Perspectives on Women, Work, and Demographic Change. Trabajo presentado en Annual World Bank Conference on Development Economics, Washington, D.C.

Gilligan, Carol (1982). In a Different Voice: Psychological Theory and Women's Development. Cambridge, MA: Harvard University Press.

Hirata, Helena; Araujo, Nadya (org.) (2012). Cuidado e cuidadoras: As várias faces do trabalho do care. São Pablo: Atlas.

Hissong, Robin (1996). Las teorías y las prácticas de desarrollo desde la perspectiva de la modernidad. Bogotá: CIDER.

Hochschild, Arlie (1983). The Managed Heart: Commercialization of Human Feeling. Berkeley: University of California Press.

Hochschild, Arlie; Machung, Anne (1990). The Second Shift. New York: Avon Books.

Kang, Miliann (2010). The Managed Hand. Race, Gender, and the Body in Beauty Service Work. Los Angeles: University of California Press.

León, Magdalena (1993). Trabajo doméstico y servicio doméstico en Colombia, en Muchacha, cachifa, criada, empleada, empregadinha, sirvienta y... más nada, trabajadoras del hogar en América Latina y el Caribe (pp. 281-302), editado por Elsa Chaney; Mary García. Caracas: Nueva Sociedad. 
Lloyd-Sherlock, Peter; Locke, Catherine (2008). Vulnerable Relations: Lifecourse, Wellbeing and Social Exclusion in a Neighbourhood of Buenos Aires, Argentina. Ageing and Society, $28(6), 779-803$.

McDowell, Linda (2009). Service Employment and the Commoditization of the Body. En Working Bodies. Interactive Service Employment and Workplace Identities (pp. 1-22). Chichester: Blackwell.

Martínez, José; Albán, Ángela; Arcos, Fabio; Calvachi, Jennyffer; Giraldo, Luisa; Jojoa, Juan (2009). Calidad de vida y adaptación social de los cuidadores de adultos mayores. Revista Colombiana de Psiquiatría, 38(4), 645-653.

Meertens, Donny (2011), La dimensión de género en el desplazamiento interno: respuestas institucionales en el caso colombiano. Revista de Derechos Humanos 9, 41-52.

Molinier, Pascale (2011). Antes que todo, el cuidado es un trabajo. En El trabajo y la ética del cuidado (pp. 15-21), editado por Luz Gabriela Arango; Pascale Molinier. Medellín: La Carreta/Universidad Nacional de Colombia.

Oakley, Ann (1974). The Sociology of Housework. London: Martin Robertson.

Paperman, Patricia (2011). La perspectiva del care: de la ética a lo político. En El trabajo y la ética del cuidado (pp. 25-44), compilado por Luz Arango; Pascale Molinier. Medellín: La Carreta/Universidad Nacional de Colombia.

Pineda, Javier (2010). Familia postmoderna popular, masculinidades y economía del cuidado. Revista Latinoamericana de Estudios de Familia, 2, 51-78.

Pineda, Javier (2011). La carga del trabajo de cuidado: distribución social y negociación familiar. En El trabajo y la ética del cuidado (pp.35-75), compilado por Luz Arango; Pascale Molinier. Medellín: La Carreta/Universidad Nacional de Colombia.

Pineda, Javier (2014). Trabajo de cuidado de la vejez en una sociedad en envejecimiento. La manzana de la discordia, 9(1), 53-69.

Pineda, Javier (2018). Cuidado institucionalizado y vejez. En Géneroy cuidado:teorías escenarios y políticas (pp. 220-241), compilado por Adira Amaya; Luz Arango; Tania Pérez-Bustos; Javier Pineda. Bogotá: Universidad Nacional de Colombia/Universidad de los Andes/ Universidad Pontificia Javeriana.

Pineda, Javier; Guevara, Nora (2014). Informe del proyecto vejez y trabajo de cuidado en una sociedad en envejecimiento. Bogotá: CIDER/Universidad de los Andes.

Posso-Quiceno, Jeanny Lucero (2011). "El proceso de socialización de la empleada doméstica: mujeres inmigrantes negras en Cali”. En El trabajo y la ética del cuidado (pp. 209-227), compilado por Luz Gabriela Arango; Pascale Molinier. Medellín: La Carreta/Universidad Nacional de Colombia. 
Razavi, Shahra (2007). The Political and Social Economy of Care in a Development Context: Conceptual Issues, Research Questions and Policy Options. En Gender and Development (pp. 1-50). Ginebra: UNRISD.

Robles, Leticia (2006). El cuidado a los ancianos: la feminización de la obligación filial. En Miradas sobre la vejez. Un enfoque antropológico (pp. 247-285), editado por Leticia Robles; Felipe Vázquez; Laureano Reyes; Imelda Orozco. México: Plaza y Valdés.

Scott, Joan (1993). "La mujer trabajadora en el siglo XIX". En Historia de las mujeres. El siglo XIX: cuerpo, trabajo y modernidad (pp. 405-436), editado por Georges Duby; Michelle Perrot. Madrid: Taurus.

Soares, Angelo (2012). As emoções do care. En Cuidado e cuidadoras: As várias faces do trabalho do care (pp. 44-59), editado por Helena Hirata; Nadia Araujo. São Pablo: Atlas.

Tronto, Joan (2013). Caring Democracy, Markets, Equality, and Justice. New York: NYU Press.

Vega, Cristina; Gutiérrez, Encarnación (2014). Nuevas aproximaciones a la organización social del cuidado: debates latinoamericanos. Presentación del dossier. Íconos, 50, 9-26.

Zambrano, Renato; Ceballos, Patricia (2007). Síndrome de carga del cuidador. Revista Colombiana de Psiquiatría, 36(1), 26-39. 